\section{(6) OPEN ACCESS}

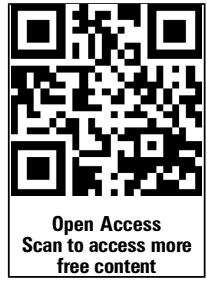

Handling editor Tore K Kvien

- Additional material is published online only. To view please visit the journal online (http://dx.doi.org/10.1136/ annrheumdis-2013-203915)

${ }^{1}$ Geriatric Research Education and Clinical Center (GRECC) Palo Alto, California, USA

${ }^{2}$ Division of Immunology and Rheumatology, Stanford University, Stanford, California, USA

${ }^{3}$ Institute for Environmental Medicine, Karolinska Institutet, Stockhoom, Sweden

${ }^{4}$ Rheumatology Unit, Department of Medicine and Center for Molecular Medicine, Karolinska Institute, Stockholm, Sweden

${ }^{5}$ Bio-Rad Laboratories, Hercules, California, USA

Correspondence to Dr William H Robinson, Division of Immunology and Rheumatology, Stanford University School of Medicine, Palo Alto VA Health Care System, 3801 Miranda Ave. Palo Alto, CA 94304, USA; wrobins@stanford.edu

Received 8 May 2013 Revised 9 September 2013 Accepted 5 November 2013 Published Online First

2 December 2013

CrossMark

To cite: Wagner CA

Sokolove J, Lahey LJ, et al. Ann Rheum Dis

2015;74:579-586.

\title{
Identification of anticitrullinated protein antibody reactivities in a subset of anti-CCP-negative rheumatoid arthritis: association with cigarette smoking and HLA-DRB1 'shared epitope' alleles
}

Catriona A Wagner, ${ }^{1,2}$ Jeremy Sokolove, ${ }^{1,2}$ Lauren J Lahey, ${ }^{1,2}$ Camilla Bengtsson, ${ }^{3}$ Saedis Saevarsdottir ${ }^{4}$ Lars Alfredsson ${ }_{1}{ }^{1}$ Michelle Delanoy, ${ }^{5}$ Tamsin M Lindstrom, ${ }^{1,2}$ Roger P Walker ${ }^{5}$ Reuven Bromberg, ${ }^{1,2}$ Piyanka E Chandra, ${ }^{1,2}$ Steven R Binder, ${ }^{5}$ Lars Klareskog, ${ }^{4}$ William H Robinson ${ }^{1,2}$

\section{ABSTRACT}

Introduction A hallmark of rheumatoid arthritis (RA) is the development of autoantibodies targeting proteins that contain citrulline. Anticitrullinated protein antibodies (ACPAs) are currently detected by the commercial cyclic citrullinated peptide (CCP) assay, which uses a mix of cyclised citrullinated peptides as an artificial mimic of the true antigen(s). To increase the sensitivity of ACPA detection and dissect ACPA specificities, we developed a multiplex assay that profiles ACPAs by measuring their reactivity to the citrullinated peptides and proteins derived from RA joint tissue.

Methods We created a bead-based, citrullinated antigen array to profile ACPAs. This custom array contains 16 citrullinated peptides and proteins detected in RA synovial tissues. We used the array to profile ACPAs in sera from a cohort of patients with RA and other non-inflammatory arthritides, as well as sera from an independent cohort of RA patients for whom data were available on carriage of HLA-DRB1 'shared epitope' (SE) alleles and history of cigarette smoking.

Results Our multiplex assay showed that at least 10\% of RA patients who tested negative in the commercial CCP assay possessed ACPAs. Carriage of HLA-DRB1 SE alleles and a history of cigarette smoking were associated with an increase in ACPA reactivity-in anti$C C P^{+}$RA and in a subset of anti-CCP- RA.

Conclusions Our multiplex assay can identify ACPApositive RA patients missed by the commercial CCP assay, thus enabling greater diagnostic sensitivity. Further, our findings suggest that cigarette smoking and possession of HLA-DRB 1 SE alleles contribute to the development of ACPAs in anti-CCP- RA.

\section{INTRODUCTION}

Rheumatoid arthritis (RA) is an autoimmune synovitis characterised by the development of autoantibodies targeting the Fc portion of immunoglobulin (rheumatoid factor (RF)) as well as autoantibodies targeting citrullinated proteins (termed anticitrullinated protein antibodies (ACPAs)). ACPAs are currently detected by the commercial cyclic citrullinated peptide (CCP) assay in which a series of cyclised, synthetic peptides derived from filaggrin and identified through screening of peptide libraries are used as surrogate antigens for the detection of autoantibodies that target citrullinemodified antigens. ${ }^{12}$ However, filaggrin and these other artificial citrullinated peptides are not expressed in synovial joints and the CCP assay may therefore fail to detect antibody reactivity to citrullinated epitopes that are not represented by these artificial mimics. A multiplex assay that encompasses the citrullinated antigens that are actually present in RA joints has the potential to improve the diagnosis of RA by enabling more comprehensive detection of ACPA responses as well as stratification of RA on the basis of the fine specificity of ACPA responses.

The clinical heterogeneity of RA, for instance in disease course and treatment response, suggests that there are distinct subtypes of RA. Therefore, there is a need for biomarker tests that can both accurately diagnose RA and molecularly stratify it. Current diagnostic tests for RA are based on single biomarkers, and their diagnostic sensitivities and specificities are limited. For example, RF has limited sensitivity and specificity for the diagnosis of RA, and autoantibodies detected by the CCP test (anti-CCP antibodies) have a specificity of up to $98 \%$ but a sensitivity of only $\sim 68 \% .^{2}$ Further, current tests cannot differentiate subtypes of ACPA-positive $\left(\mathrm{ACPA}^{+}\right)$RA. We previously demonstrated that analysis of multiple biomarkers can delineate RA subtypes on the basis of the fine specificity of ACPA responses and can provide insight into clinically identifiable RA subtypes such as disease initiation or severity. ${ }^{3}{ }^{4}$ Recent findings showed that a history of cigarette smoking is an environmental risk factor for RA, but only in individuals who carry HLA-DRB1 shared epitope (SE) alleles and are anti-CCP-positive (anti-CCP $\left.{ }^{+}\right),{ }^{5-8}$ underscoring the value of accurately identifying individuals with ACPA reactivity.

Citrullination is the post-translational modification of arginine to citrulline by peptidyl arginine demininase. A variety of proteins present in synovial tissues become citrullinated in the inflamed RA joint. To better define the specific citrullinated proteins present in RA joint tissues, we and others have performed proteomic analysis of synovial 
proteins targeted by autoantibodies present in the blood of RA patients. ${ }^{910}$ Using the citrullinated and other proteins and peptides identified by these analyses, we previously developed a planar, research-grade array containing $>200$ putative RA autoantigens and used it to identify serum autoantibody profiles associated with clinical subtypes of RA. ${ }^{3} 4$

In this study, we aimed to develop a reproducible assay for ACPA profiling that is amenable to automation and, ultimately, to use in clinical care. We created a multiplex, bead-based antigen array by transferring select citrullinated antigens identified previously ${ }^{3} 10$ onto the Luminex 200 running Bio-Plex Software V.5.0. We then used this custom array to profile ACPAs in sera derived from patients with RA, other arthritides and healthy controls. We detected ACPAs in at least $10 \%$ RA patients who were anti-CCP ${ }^{-}$in the Stanford Rheumatic Disease Registry Cohort, and then validated and extended this result in the Epidemiological Investigation of RA (EIRA) cohort. Thus, we identified a panel of citrullinated antigens that provide improved detection of ACPAs, and using samples from the EIRA cohort found that the combination of cigarette smoking and carriage of the HLA-DRB1 SE alleles is associated with greater ACPA reactivity in anti-CCP ${ }^{-}$RA patients.

\section{MATERIALS AND METHODS}

\section{Samples}

All serum samples were collected after receipt of informed patient consent at Stanford University or the Karolinska Institute under protocols approved by the Institutional Review Board at Stanford University or the Karolinska Institute, respectively. The samples collected at Stanford University were provided by the Stanford Rheumatic Disease Registry and include sera from 30 patients with established RA, 23 patients with systemic lupus erythematosus (SLE), nine patients with psoriatic arthritis (PsA), six patients with gout and 10 healthy individuals. Stanford Rheumatic Disease Registry samples were collected and processed using uniform protocols, stored in the same freezer and results are from the first thaw of the sample. The samples provided by the Karolinska Institute were collected as part of the EIRA, a Swedish population-based case-control study, which enrols newly diagnosed individuals aged 18-70 who were recruited from May 1996 until May 2006 from a geographically defined region in Sweden. ${ }^{11}$ Anti-CCP antibody status was analysed using a CCP2 assay (EIRA: Eurodiagnostica; Stanford Rheumatic Disease Registry: Axis-Shield) according to the manufacturer's instructions. For both cohorts, the diagnosis of RA was made according to the American College of Rheumatology 1987 criteria. $^{12}$

\section{Multiplex autoantibody assays}

We developed a custom, bead-based, antigen array on the Luminex 200 running Bio-Plex Software 5.0 for measurement of ACPAs targeting 16 putative RA-associated autoantigens (see online supplementary table S1). As described in figure 1, carboxyl groups bound to spectrally distinct beads were activated with 1-ethyl-3-[3-dimethylaminopropyl]carbodiimide hydrochloride and $N$-hydroxysulfosuccinimide. These ester groups bound directly to the primary amines of antigen protein or streptavidin. Peptides were synthesised with an amine-terminus biotin followed by a hydrophilic aminohexanoic acid spacer and coupled to streptavidin-coated beads. Antigen-coated beads were washed with a Bio-Plex Pro II Washer, pooled and incubated with serum samples (1:30 dilution) at room temperature. After three washes, antihuman IgG antibody conjugated to phycoerythrin (PE) was incubated with the beads at room temperature. After an additional three washes, beads were resuspended in buffer and analysed on a Luminex 200 running Bio-Plex Software V.5.0 (Bio-Rad, Hercules, California, USA) for determination of PE fluorescence and bead identity.

\section{Statistical analysis}

To identify significant differences in antibody-antigen reactivity between groups, we analysed the fluorescence readouts by significance analysis of microarrays $(\mathrm{SAM})^{13}$ and sorted the SAM results on the basis of false discovery rates. We used hierarchical clustering software (Cluster 3.0) to arrange SAM results according to degree of similarity among patient samples in autoantibody reactivities, and Java Treeview ${ }^{14}$ software to graphically display the results as a heatmap. Based on the SAM results, we identified ACPAs that could distinguish between RA patients and controls by comparing the mean fluorescence readout of reactivity for each antigen between RA cases and controls. We used these differentiating ACPAs in our final analysis aimed at evaluating the sensitivity and specificity of the multiplex assay in diagnosing RA.

A patient sample was deemed to contain antibodies to a specific antigen on the array (ie, to be 'positive' for the ACPA recognising that antigen) if the mean of the corresponding fluorescence value was three times that of the mean fluorescence value for the same antigen in samples from healthy individuals. An 'ACPA score' for each sample was determined by dividing the fluorescent value for each ACPA reactivity by the mean value for that specific ACPA and then taking the sum ( $\Sigma$ (ACPA value/mean ACPA value)). We used one-way analysis of variance and Dunnett's post hoc test (Graph-Pad Prism 5) to analyse the differences in ACPA score and in number of positive (antigenbinding) ACPAs between patients with differing smoker, HLA-DRB1 SE alleles and anti-CCP antibody status. p Values less than 0.05 were considered significant.

The reproducibility of the bead-based multiplex array was determined by establishing inter-assay and intra-assay coefficients of variance $(\mathrm{CVs})$. Intra-assay reproducibility was determined by analysing 10 replicates of four samples with low, medium or high ACPA reactivity within a single run. Inter-assay CVs were determined by running three samples with no, low or high ACPA reactivity in 10 independent runs.

\section{RESULTS}

\section{Development of a multiplexed, bead-based, antigen array for profiling ACPAs in RA}

We sought to develop a multiplex biomarker test based on ACPA profiling that could ultimately be used in clinical care. We transferred the most promising of the candidate citrullinated epitopes identified previously ${ }^{4}{ }^{9} 10$ onto the Luminex 200 running Bio-Plex Software V.5.0, a multiplex, bead-based, immunoassay platform. The methods and principles of our multiplex, beadbased, antigen array are shown in figure 1 . To assess the reproducibility of our multiplex assay, we subjected it to replicate evaluation using samples from the Stanford Rheumatic Disease Registry. Intra-assay reproducibility was determined by analysing 10 replicates of each of four samples within a single run; the intra-assay CVs ranged from $0.4 \%$ to $5.5 \%$ (see online supplementary table S2). Inter-assay reproducibility was determined by analysing three samples in six or 10 independent runs; the interassay CVs ranged from $0.9 \%$ to $10 \%$ (see online supplementary table S2). These results are comparable with CVs obtained with commercially available RF and CCP ELISA tests. CCP ELISAs have intra-assay CVs ranging from $4.8 \%$ to $13 \%$ and inter-assay CVs ranging from $9 \%$ to $17 \%,{ }^{15}$ while RF ELISAs have an intra-assay CV of $6 \%$ and an inter-assay CV of $8 \% .^{16}$ 


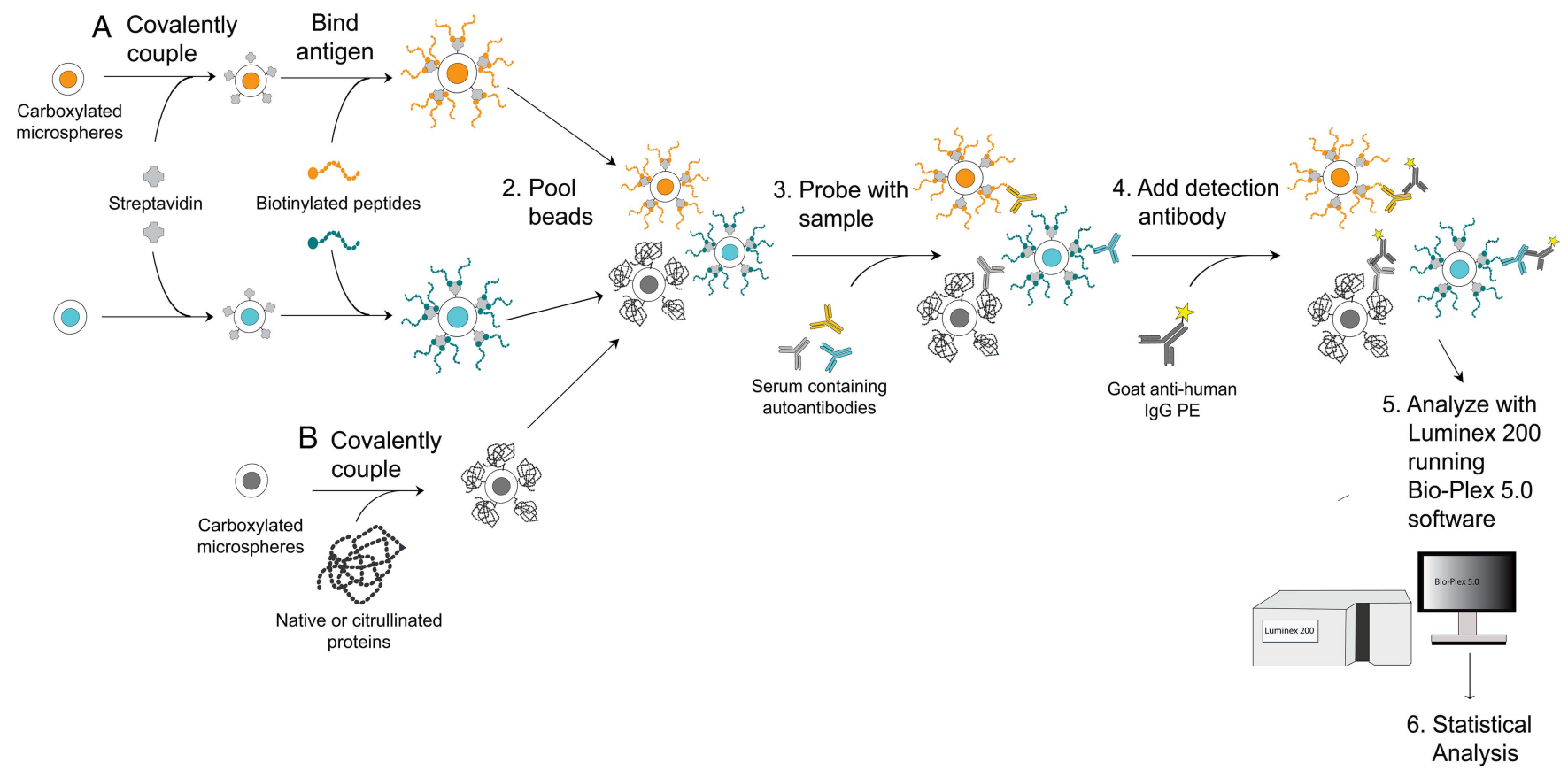

Figure 1 Schematic representation of multiplex, bead-based detection of rheumatoid arthritis-associated autoantibodies. This technology uses carboxylated beads with magnetic cores that have been dyed with spectrally distinct dye mixtures (represented as blue, grey and yellow). (1A) For analysis of autoantibodies against peptides, streptavidin is covalently bound to the carboxyl groups on the bead surface by amine coupling using 1-ethyl-3-[3-dimethylaminopropyl]carbodiimide hydrochloride (EDAC) and N-hydroxysulfosuccinimide (S-NHS). Peptides synthesised with an $\mathrm{N}$-terminal biotin are then incubated with the beads, resulting in the generation of peptide-coupled beads. (1B) For analysis of autoantibodies against proteins, native or citrullinated proteins are covalently bound to the carboxyl groups on the bead surface by amine coupling using EDAC and S-NHS. (2) Spectrally distinct beads bound to different antigens are then pooled. (3) Beads are incubated with diluted serum or plasma samples, allowing autoantibodies in the sample to bind to their cognate antigens on the beads' surface. (4) Bound antibodies are detected by using antihuman IgG conjugated to phycoerythrin (PE). (5) A Luminex 200 running Bio-Plex Software V.5.0 is used for analysis of (i) the dye mixture of the bead and thereby identification of the bound antigen and (ii) the PE fluorescence and thereby the level of autoantibody binding. (6) Statistical analysis is performed.

We used our antigen array to analyse ACPAs in sera from 30 patients with RA, 23 patients with SLE, nine patients with PsA, six patients with gout and 10 healthy individuals (table 1). To

Table 1 Clinical characteristics of patients with rheumatoid arthritis (RA) or other non-inflammatory arthritides (non-RA) for Stanford Registry and EIRA cohorts

\begin{tabular}{|c|c|c|c|}
\hline & \multicolumn{2}{|l|}{ Stanford Registry } & \multirow{2}{*}{$\begin{array}{l}\text { EIRA } \\
\text { RA }(n=2233)\end{array}$} \\
\hline & $R A(n=30)$ & $\begin{array}{l}\text { non-RA } \\
(n=48)\end{array}$ & \\
\hline Age, mean (range) years & $46(21-65)$ & $34(19-64)$ & $51(18-70$ \\
\hline Female, no. (\%) & $26(86.7)$ & $33(86.8)$ & $1414(71)$ \\
\hline $\begin{array}{l}\text { Disease duration, mean } \\
\text { (range) years }\end{array}$ & $10.9(2.6-38.6)$ & $8.5(1.7-31.6)$ & $0 *$ \\
\hline RF-positive, no. (\%) & $11(40.7)$ & ND & $1320(66.5)$ \\
\hline CCP-positive, no. (\%) & $14(46.7)$ & ND & $1240(63.1)$ \\
\hline ESR, median(range) $\mathrm{mm} \mathrm{Hg}$ & $20(2-53)$ & ND & $26(1-130)$ \\
\hline $\mathrm{CRP}$, median (range) $\mathrm{mg} / \mathrm{dL}$ & $0.2(0.2-4.4)$ & ND & $16(0-276)$ \\
\hline $\begin{array}{l}\text { DAS28-ESR, median } \\
\text { (range) }\end{array}$ & $4.42(0.65-7.15)$ & ND & $5.32(0.54-8.89)$ \\
\hline $\begin{array}{l}\text { DAS28-CRP, median } \\
\text { (range) }\end{array}$ & $3.64(1.1-6.2)$ & ND & ND \\
\hline
\end{tabular}

determine which of the citrullinated antigens on our array are targeted by ACPAs that are specific to RA, we compared ACPA antigen reactivity in sera from individuals with RA with that in sera from individuals with SLE, PsA or gout. Among the 16 citrullinated antigens on the array, SAM analysis identified eight that were preferentially targeted by ACPA to a significantly greater extent in sera from RA patients than in sera from patients with other inflammatory arthritides (figure 2A). These targeted antigens included citrullinated peptides derived from clusterin, histone $2 \mathrm{a}$, biglycan, apolipoprotein $\mathrm{E}$, filaggrin and vimentin, as well as citrullinated forms of vimentin proteins.

All of the sera from anti-CCP ${ }^{+}$RA patients were highly reactive to the citrullinated antigens on the array, whereas most of the sera from anti-CCP ${ }^{-}$RA patients were unreactive (figure $2 \mathrm{~A})$. However, sera from two of the 16 anti-CCP ${ }^{-} \mathrm{RA}$ patients were highly reactive to most of the citrullinated antigens. In addition, serum from a third anti-CCP ${ }^{-}$RA patient (BBS113) was highly reactive to a small subset of citrullinated antigens. These findings indicate that some RA patients have ACPA antibodies despite testing negative in the commercial CCP assay.

To confirm that the reactivity detected is citrulline specific, we analysed four non-citrullinated protein controls: apolipoprotein $\mathrm{E}$, histone $2 \mathrm{~B}$, vimentin and fibrinogen. No $\mathrm{CCP}^{+} \mathrm{RA}$ patients who exhibited positive reactivity to citrullinated apolipoprotein $\mathrm{E}$ or fibrinogen A were reactive with the non-citrullinated forms of the same proteins (see online supplementary table S5). Two out of the $14 \mathrm{CCP}^{+} \mathrm{RA}$ patients who possessed autoantibody reactivity to citrullinated vimentin also exhibited reactivity to 
A

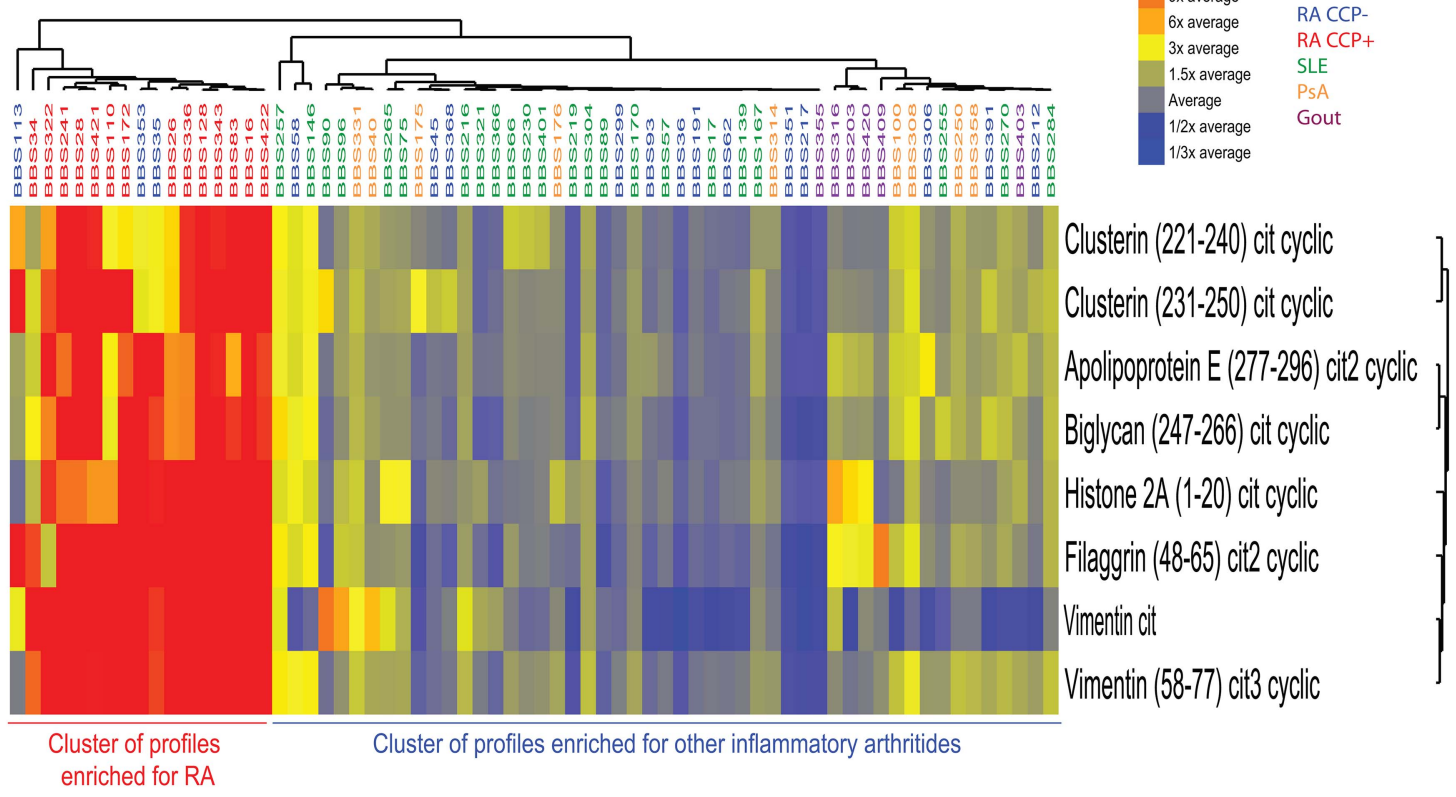

B
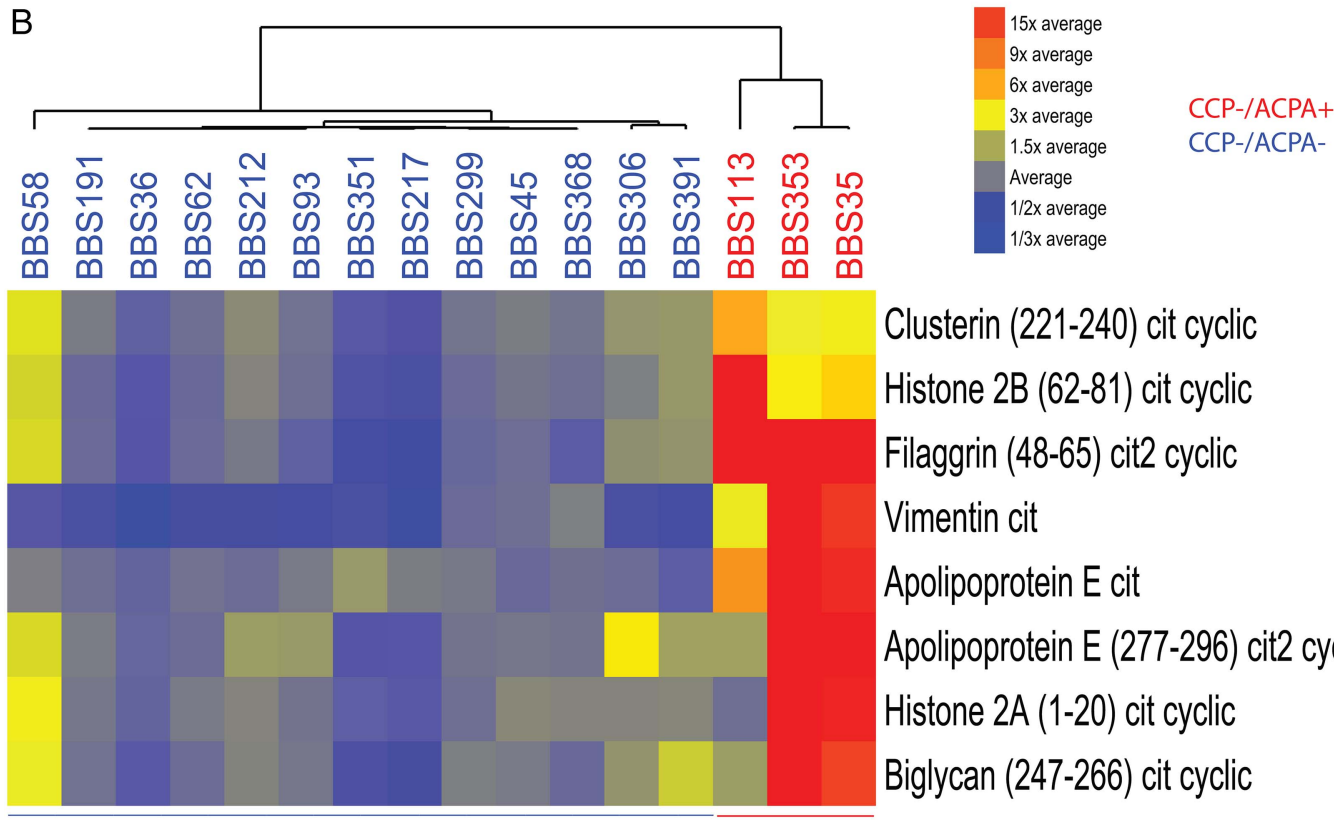

Clusterin (221-240) cit cyclic
Histone 2B (62-81) cit cyclic
Filaggrin (48-65) cit2 cyclic

Vimentin cit

Apolipoprotein $\mathrm{E}$ cit

Apolipoprotein E (277-296) cit2 cyclic

Histone $2 \mathrm{~A}(1-20)$ cit cyclic

Biglycan (247-266) cit cyclic

Cluster of profiles enriched for CCP-/ACPA-

CCP-/ACPA+

Figure 2 Stratification of rheumatoid arthritis (RA) according to the presence and specificity of anticitrullinated protein antibodies (ACPAs). The multiplex antigen array described in figure 1 was used for profiling ACPAs in (A) 16 patients with anti-CCP- ${ }^{-}$A, 14 patients with anti-CCP ${ }^{+}$RA, 23 patients with systemic lupus erythematosus (SLE), nine patients with psoriatic arthritis ( $P s A)$, six patients with gout and 10 healthy individuals, and (B) anti-CCP ${ }^{-}$RA patients identified by table 3 as $\mathrm{ACPA}^{+}$with anti-CCP- $/ \mathrm{ACPA}^{-}$RA patients. Significance analysis of microarrays was used to analyse differences between each group. Each antigen identified had a false discovery rate of $<0.1 \%$. Data were normalised as described in the Materials and methods section. A hierarchical clustering algorithm was used to determine cluster relations that group patient samples (top dendrogram) and antigen reactivities (right dendrogram) on the basis of similarities in patients' ACPA profiles. Dendrogram branch lengths and distances between nodes illustrate the extent of similarities in antigen reactivity, with blue representing a decrease relative to the mean value obtained in samples from healthy individuals, yellow no change and red an increase. CCP, cyclic citrullinated peptide; Cit, citrullinated.

the non-citrullinated form, and of the $14 \mathrm{CCP}^{+} \mathrm{RA}$ patients who possessed autoantibody reactivity to citrullinated histone $2 \mathrm{~B}$ two different patients exhibited reactivity to the noncitrullinated form. None of the $\mathrm{CCP}^{-} \mathrm{RA}$ patients who showed positive reactivity to the citrullinated forms of these proteins reacted to the non-citrullinated forms, demonstrating that these patients do have ACPAs (see online supplementary table S6).

\section{Diagnostic value of ACPA biomarker panel: identification of ACPA reactivity in $10+\%$ of $\mathrm{CCP}^{-}$samples}

To determine the diagnostic potential of the panel of 16 citrullinated antigens, we compared the diagnostic sensitivity and specificity of ACPA reactivity of different combinations of the autoantigens with that of ACPA reactivity measured by the CCP ELISA assay. Screening sera from RA, SLE, PsA and gout 
Table 2 Sensitivity and specificity analysis of select biomarker panel in diagnosing RA

\begin{tabular}{lllll}
\hline $\begin{array}{l}\text { Number of positive } \\
\text { biomarkers* }\end{array}$ & $\begin{array}{l}\text { Sensitivity } \\
(\%)\end{array}$ & $\begin{array}{l}\text { Specificity } \\
(\%)\end{array}$ & $\begin{array}{l}\text { PPV } \\
(\%)\end{array}$ & $\begin{array}{l}\text { NPV } \\
(\%)\end{array}$ \\
\hline$\geq 1$ markers & 70 & 50 & 47 & 73 \\
$\geq 2$ markers & 60 & 85 & 72 & 77 \\
$\geq 3$ markers & 57 & 92 & 81 & 77 \\
$\geq 4$ markers & 57 & 96 & 90 & 78 \\
$\geq 5$ markers & 57 & 96 & 90 & 78 \\
CCP2 ELISA & 47 & ND & ND & ND \\
\hline
\end{tabular}

Sera analysed PPVs were from patients with RA $(n=30)$, SLE $(n=23), \operatorname{PsA}(n=9)$, gout $(n=6)$ and healthy controls $(n=10)$.

*Biomarker panel identified in online supplementary table $\mathrm{S1}$.

CCP, cyclic citrullinated peptide; ND, not determined; NPV, negative predictive value; PPV, positive predictive value; PSA, psoriatic arthritis; RA, rheumatoid arthritis; SLE, systemic lupus erythematosus.

patients and from healthy controls, we evaluated the performance of reactivity to one to five of the 16 autoantigens in the differential diagnosis of RA. Reactivity to any four of the 16 antigens was optimal, yielding a specificity of $96 \%$ and a sensitivity of $57 \%$ for the diagnosis of RA (table 2). This specificity is comparable with that reported for the commercial CCP assay. $^{2}$ The sensitivity of our assay is greater than the $46.7 \%$ sensitivity achieved by the commercial CCP assay in the same patient cohort (table 2), enabling detection of ACPAs in 18.8\% (three out of 16) of the RA patients who tested negative for ACPAs in the CCP assay (ie, anti-CCP ${ }^{-}$patients) (table 3). We validated this result using 249 healthy controls and 1983 RA patients from the EIRA cohort. In this analysis, reactivity to any two of the 16 antigens was optimal, yielding a sensitivity of $63 \%$ and a specificity of $98 \%$ (see online supplementary table S3). Using the optimal cut-off, $10 \%$ (73 out of 733) of the RA patients in the EIRA cohort who tested $\mathrm{CCP}^{-}$were found to be $\mathrm{ACPA}^{+}$(see online supplementary table S4).

To gain insights into the citrullinated antigens targeted in anti-CCP ${ }^{-}$but $\mathrm{ACPA}^{+}$RA patients, we used SAM to compare ACPA reactivities in anti-CCP ${ }^{-} / \mathrm{ACPA}^{+}$patients and anti-CCP ${ }^{-} /$ $\mathrm{ACPA}^{-}$patients. SAM analysis showed that ACPA reactivity to citrullinated forms of apolipoprotein $\mathrm{E}$ and vimentin, as well as peptides derived from citrullinated clusterin, filaggrin, histone $2 \mathrm{~B}$, histone $2 \mathrm{~A}$, biglycan and apolipoprotein $\mathrm{E}$, was significantly

Table 3 Proportion of RA patients and controls identified as $\mathrm{ACPA}^{+}$by the ACPA biomarker panel ${ }^{*}$

\begin{tabular}{lllll}
\hline & $\begin{array}{l}\text { Total } \\
\text { number of } \\
\text { individuals }\end{array}$ & $\begin{array}{l}\text { Number of } \\
\text { ACPA }^{+} \\
\text {individuals }\end{array}$ & $\begin{array}{l}\text { Number of } \\
\text { ACPA }^{-} \\
\text {individuals }\end{array}$ & $\begin{array}{l}\text { \% of } \text { ACPA }^{+} \\
\text {individuals }\end{array}$ \\
\hline $\begin{array}{c}\text { RA patients } \\
\text { CCP }^{-}\end{array}$ & 30 & 17 & 13 & 56.7 \\
CCP $^{+}$ & 16 & 3 & 13 & 18.8 \\
Controls & 14 & 14 & 0 & 100 \\
Healthy & 48 & 2 & 46 & 4.2 \\
SLE & 10 & 0 & 10 & 0 \\
Gout & 23 & 2 & 21 & 8.7 \\
PsA & 6 & 0 & 6 & 0 \\
\hline
\end{tabular}

*ACPA positivity was defined as mean reactivity being three times that for the same antigen in samples from healthy controls. Individuals were considered $\mathrm{ACPA}^{+}$if they were positive for $\geq 4$ of the 16 RA-associated ACPAs listed in online supplementary table $\mathrm{S1}$

ACPA, anticitrullinated protein antibody; CCP, cyclic citrullinated peptide; PsA, psoriatic arthritis; RA, rheumatoid arthritis; SLE, systemic lupus erythematosus. higher in the anti-CCP${ }^{-} / \mathrm{ACPA}^{+}$group than in the anti-CCP${ }^{-}$/ $\mathrm{ACPA}^{-}$group. Hierarchical clustering of patient samples on the basis of their autoantibody reactivities showed the anti-CCP${ }^{-}$/ $\mathrm{ACPA}^{+}$and anti-CCP$-/ \mathrm{ACPA}^{-}$patients group into two distinct clusters based on ACPA reactivity (figure $2 \mathrm{~B}$ ).

\section{High ACPA reactivity is associated with smoking and carriage of $H L A-D R B 1$ SE alleles in both anti-CCP ${ }^{+}$ and anti-CCP ${ }^{-}$RA}

Klareskog $e t a l^{8}$ recently showed that cigarette smoking and carriage of HLA-DRB1 SE alleles are both risk factors for RA, but only anti-CCP ${ }^{+}$RA. Moreover, they uncovered a marked interaction between these two risk factors. The relative risk for RA was 21-fold higher in smokers with both copies of the HLA-DRB1 SE alleles than in non-smokers with no HLA-DRB1 SE alleles; again, this effect was limited to anti-CCP ${ }^{+}$RA. To determine whether we could recapitulate these findings with our 16 citrullinated antigen panel, using samples derived from the EIRA cohort we analysed ACPA reactivity in anti-CCP ${ }^{+}$and anti-CCP ${ }^{-}$RA patients including smokers and non-smokers with or without HLA-DRB1 SE alleles (table 1).

Among anti-CCP ${ }^{+} \mathrm{RA}$ patients, carriage of one or two HLA-DRB1 SE alleles or a combination of both smoking and HLA-DRB1 SE alleles was associated with an increase of number of ACPAs (figure 3A,D). Likewise, in anti-CCP ${ }^{+} \mathrm{RA}$ patients, smoking, carriage of HLA-DRB1 SE alleles or a combination of both was associated with an increase in titre of ACPAs (figure $3 \mathrm{~A}, \mathrm{D})$. There was also an increase in number and titre of ACPA between patients who only smoked or who possessed HLA-DRB1 SE alleles and those who had both risk factors (figure $3 \mathrm{~A}, \mathrm{D}$ ).

Among the anti-CCP ${ }^{-}$patients, the combination of smoking and carriage of HLA-DRB1 SE alleles was associated with an increase in number of ACPAs (figure 3B,D). An analysis of the top 50th percentile of anti-CCP ${ }^{-}$patients was performed to eliminate the patients who are truly $\mathrm{ACPA}^{-}$, thereby enabling focused analysis of the anti-CCP${ }^{-} \mathrm{RA}$ patients exhibiting the highest ACPA levels. In this subset of anti-CCP ${ }^{-}$patients, the combination of smoking and carriage of HLA-DRB1 SE alleles was associated with an increase in number and titre of ACPAs (figure 3C,D). The combination of smoking and HLA-DRB1 SE alleles was associated with higher ACPA titre as compared with those who had a history of smoking but did not possess the HLA-DRB1 SE alleles (figure 3C,D). Thus, the combination of smoking and carriage of HLA-DRB1 SE alleles is associated with the presence of ACPA in anti-CCP ${ }^{-}$RA patients.

\section{DISCUSSION}

In this study, we describe the development of a bead-based, multiplex, antigen array for profiling ACPAs in RA. Using this assay, we identified ACPA profiles that distinguished patients with RA from those with other inflammatory arthritides. We also used this assay in uncovering a subset of anti-CCP ${ }^{-}$RA patients who have ACPAs, and in confirming that smoking and carriage of the HLA-DRB1 SE are associated with greater ACPA reactivity.

ACPAs are a hallmark of RA, and their presence is one of the criteria for the diagnosis of RA (though approximately a third of RA patients never develop ACPAs). Currently, ACPAs are detected by the CCP assay, which measures ACPA reactivity to synthetic mimics of the citrullinated antigens targeted in RA. Although the CCP assay is highly specific in diagnosing RA, it is not very sensitive. As $\mathrm{we}^{3}$ and others ${ }^{2}$ have proposed, profiling ACPA reactivity to a panel of citrullinated antigens that are 

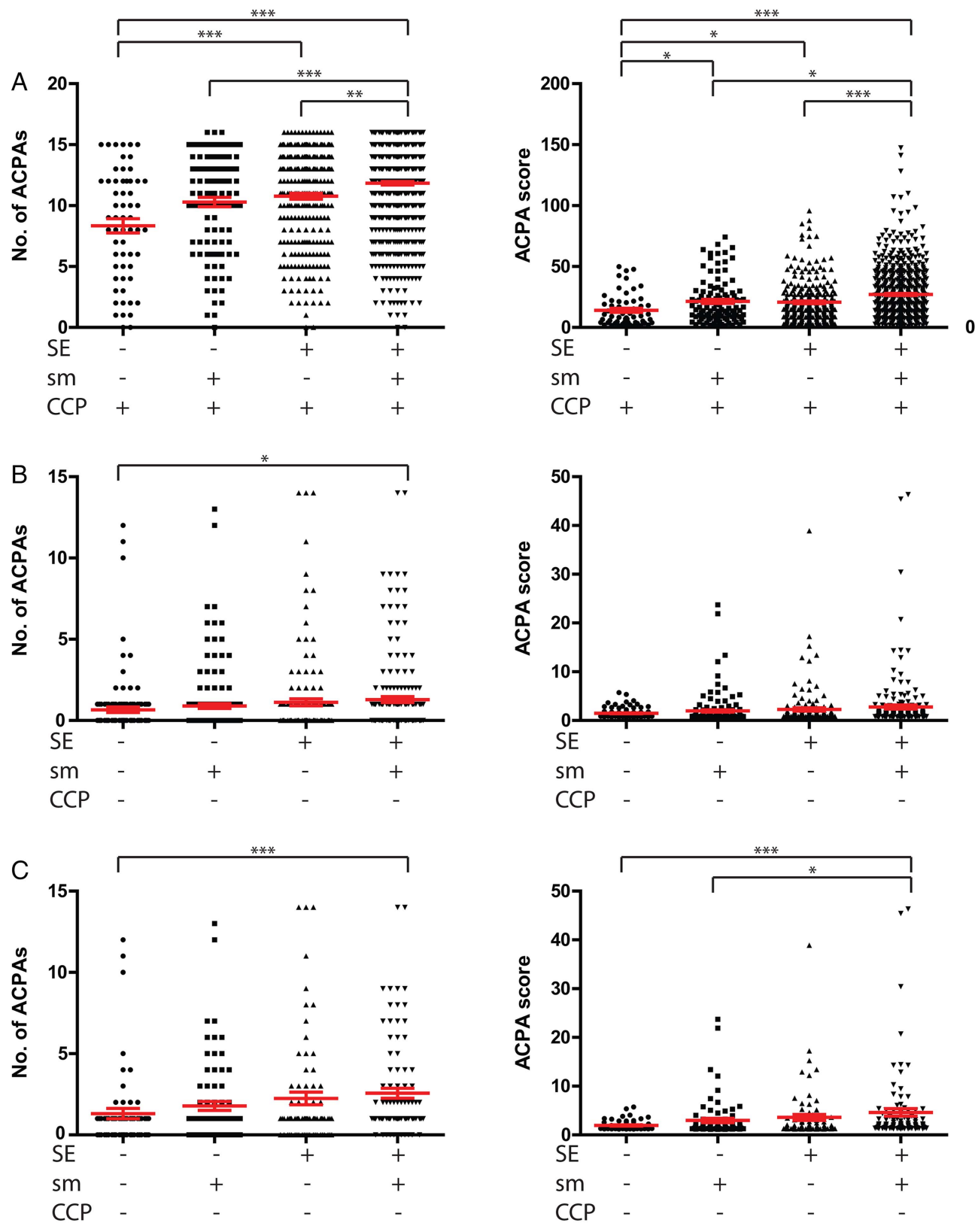

D

\begin{tabular}{|c|c|c|c|c|c|c|}
\hline \multirow{2}{*}{ Comparison } & \multicolumn{2}{|c|}{ CCP+ } & \multicolumn{2}{c|}{ CCP- (All) } & \multicolumn{2}{c|}{ CCP- (Top 50\%) } \\
\cline { 2 - 7 } & $\begin{array}{c}\text { No. of ACPAs } \\
P \text { value }\end{array}$ & $\begin{array}{c}\text { ACPA score } \\
P \text { value }\end{array}$ & $\begin{array}{c}\text { No. of ACPAs } \\
P \text { value }\end{array}$ & $\begin{array}{c}\text { ACPA score } \\
P \text { value }\end{array}$ & $\begin{array}{c}\text { No. of ACPAs } \\
P \text { value }\end{array}$ & $\begin{array}{c}\text { ACPA score } \\
P \text { value }\end{array}$ \\
\hline SE-sm- vs SE-sm+ & ns & $<0.05$ & $n s$ & $n s$ & $n s$ & $n s$ \\
SE-sm- vs SE+sm- & $<0.001$ & $<0.05$ & $n s$ & $n s$ & $n s$ & $n s$ \\
SE-sm- vs SE+sm+ & $<0.001$ & $<0.001$ & $<0.05$ & $n s$ & $<0.001$ & $<0.001$ \\
SE-sm+ vs SE+sm+ & $<0.001$ & $<0.05$ & $n s$ & $n s$ & $n s$ & $<0.01$ \\
SE+sm- vs SE+sm+ & $<0.01$ & $<0.001$ & $n s$ & $n s$ & $n s$ & $n s$ \\
SE-sm+ vs SE+sm- & $n s$ & $n n$ & $n s$ & $n s$ & $n s$ & $n s$ \\
\hline
\end{tabular}

Figure 3 Anticitrullinated protein antibody (ACPA) reactivity in rheumatoid arthritis (RA) analysed by smoking status and possession of HLA-DRB1 shared epitope (SE) alleles. Using samples from the Epidemiological Investigation of RA cohort, the number of ACPAs and the ACPA score were determined for the following patient subsets: (A) anti-CCP+ ${ }^{+}$RA patients, (B) anti-CCP ${ }^{-}$RA patients and (C) the top 50th percentile of anti-CCP patients (based on ACPA score). The results are grouped by smoking and SE status: $\mathrm{SE}^{+} \mathrm{sm}^{+} C C P^{+}(\mathrm{n}=644), \mathrm{SE}^{+} \mathrm{sm}^{-} \mathrm{CCP}^{+}(\mathrm{n}=269), \mathrm{SE}^{-} \mathrm{sm}^{+} C C P^{+}$

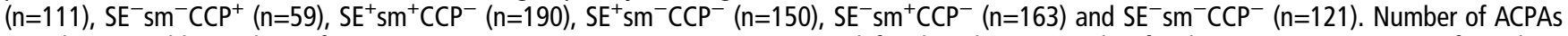
was determined by analysis of reactivity to 16 antigens; positive reactivity was defined as three times that for the same antigen in sera from the healthy controls. ACPA score is determined for each sample by dividing each ACPA value by the mean ACPA value and taking the sum of this product for each ACPA. ${ }^{*} p \leq 0.05,{ }^{* *} p \leq 0.01$ and ${ }^{* * *} p \leq 0.001$ by one-way analysis of variance (ANOVA) and Dunnett's post hoc test. (D) ANOVA and Dunnett's post hoc test results for each combination of smoking, HLA-DRB1 SE alleles and anti-CCP status. CCP, cyclic citrullinated peptide. 
actually present in RA synovial joints could improve the sensitivity of diagnosing RA. Although SAM identified eight antigens as significantly different between the group of RA patients and with other diseases, at the patient-by-patient level some of the RA patients exhibited reactivity to other citrullinated antigens not identified by SAM. Therefore, we used the entire biomarker panel for sensitivity and specificity analysis.

Indeed, the biomarker panel of 16 citrullinated autoantigens identified RA patients with a sensitivity greater than that achieved by the commercial CCP assay in the same cohort. Furthermore, the specificity of this biomarker panel in diagnosing RA was $96 \%$, a specificity comparable with that of the CCP assay. $^{2}$ Only two out of 48 controls were identified as $\mathrm{ACPA}^{+}$ using our panel and these controls were both SLE patients. Although the clinical data did not include CCP ELISA reactivity for control samples, we analysed reactivity to a CCP bead in our assay. When using a cut-off of three times higher than the average reactivity of healthy controls to determine positivity, both SLE samples that were positive in the multiplex assay are CCP-negative.

The peptides used in the CCP assay are derived from filaggrin and other peptides identified in phage display screens. ${ }^{1} 2$ They are an artificial mimic of the citrullinated antigens present in the RA joint, and thus may not fully represent the spectrum of citrullinated epitopes present in and targeted by autoantibody responses in RA. While this may not be a problem if ACPA reactivity is directed solely against epitopes present on citrulline itself, evidence suggests that ACPA reactivity depends in part on the amino acids flanking citrulline. ${ }^{17}$ Therefore, optimal diagnosis of RA most likely requires assessment of ACPA reactivity to the specific citrullinated antigens targeted in RA. By including in our antigen array citrullinated peptides derived from proteins thought to be present in RA joints, our assay covers a greater number of putative RA-associated autoantigens and was thus able to detect the presence of ACPAs in RA patients who tested negative in the CCP assay.

Although the peptides comprising the CCP assay (the CCP2 assay) used may mimic several different citrullinated antigens, the sequence of these peptides remains undisclosed. Further, despite detecting ACPAs with great sensitivity, the CCP assay does not identify the specific antigens targeted by the ACPAs; therefore, it does not provide insight into the mechanisms underlying RA. By contrast, our assay reveals ACPA targeting of specific, citrullinated, pathologically relevant antigens that may play unique roles in the initiation and/or propagation of RA.

Next-generation CCP tests are being developed, and recently the CCP3 and anti-modified citrullinated vimentin (MCV) tests were described. For our studies, we used the CCP2 assay because of its widespread use in clinical practice. There has also been no significant improvements shown in sensitivity or specificity between CCP2 and either CCP3 ${ }^{18} 19$ or anti-MCV assays. ${ }^{20}$ As a result, we can infer that our assay will have a higher sensitivity than both the CCP3 and anti-MCV assays, although further analysis will need to be performed.

RA is a heterogeneous disease varying in clinical features, disease severity and response to treatment. Currently, the field acknowledges the existence of two subsets of RA: anti-CCP ${ }^{+}$ and anti-CCP ${ }^{-} \mathrm{RA},{ }^{21}$ subsets that are thought to reflect the presence or absence of ACPAs. However, our multiplex assay revealed that three out of 16 anti-CCP ${ }^{-}$RA samples we analysed did in fact have ACPAs. ACPAs may develop before the onset of clinical $\mathrm{RA}^{22} 23$ and are associated with more severe and erosive disease, including bone erosion. ${ }^{24-26}$ Recently, a study demonstrated that methotrexate therapy during undifferentiated arthritis delayed progression to RA, but only in the patients who had ACPA reactivity as defined by the CCP test. ${ }^{27}$ There is therefore a need for a diagnostic test that can reliably identify, at an early stage, patients who have ACPAs. If these patients go undetected, owing to lack of sensitivity of the CCP assay, they may not receive potentially beneficial therapy. Thus, use of an ACPA-profiling assay could enable more accurate classification of RA subsets and identify those likely to respond to specific therapies. Additionally, the ability to directly evaluate ACPA fine specificity in a high-throughput and highly reproducible assay could enable the identification of molecular subtypes of $\mathrm{ACPA}^{+}$ $\mathrm{RA}$, and hence the prediction of disease outcome and response to therapy.

Although the aetiology of RA is unknown, recent findings suggest that smoking acts as an environmental trigger of RA by inducing anticitrulline immunity in individuals possessing HLA-DRB1 SE alleles. ${ }^{8}$ The findings from our ACPA-profiling assay support this hypothesis, confirming that among anti-CCP ${ }^{+}$ RA patients, smoking and carriage of the HLA-DRB1 SE alleles are associated with greater ACPA reactivity. Moreover, we show that this is also the case in a subset of patients with anti-CCP RA. Our findings indicate that a subset of anti-CCP ${ }^{-}$patients actually do possess ACPAs, highlighting the need for nextgeneration diagnostics to detect ACPAs in these patients. Further, our results confirm the interaction between the HLA-DRB1 SE alleles and smoking in anti-CCP ${ }^{+} \mathrm{RA}$ patients, and suggest that cigarette smoking and the HLA-DRB1 SE alleles may contribute to the development of ACPA in anti-CCP ${ }^{-}$RA patients.

This study has several limitations. Because its aim was to provide proof of concept that our novel ACPA-profiling array could detect pathobiologically and clinically relevant ACPA profiles, the sample size analysed is small and our findings therefore remain to be validated in a larger, independent cohort. We were also unable to adjust for treatment-related effects that could affect the levels of ACPAs and therefore confound interpretation of our data. Although efforts were made to match patient groups based on disease duration, sex, age and other demographic parameters, when such parameters were available, differences in the underlying demographics of the groups could also confound the results. Additionally, although our assay includes putative autoantigens identified through proteomic screening, ${ }^{9}$ there are likely additional citrullinated antigens present in RA joints. This provides the potential for even further expansion of the antigens represented on our array, with an eye toward further increasing the sensitivity of ACPA profiles in diagnosing RA, as well as further delineating molecular subtypes of clinical significance.

\section{CONCLUSIONS}

In this study, we describe the development and application of a multiplex antigen array for profiling ACPAs in RA. The ACPA profiles we identified with our array had comparable specificity and greater sensitivity than the commercial CCP test in diagnosing RA. Indeed, our array enabled the detection of ACPAs in at least $10 \%$ of RA patients who tested negative for ACPA reactivity in the CCP2 assay. Although these ACPA profiles remain to be validated as biomarkers of RA, our findings suggest that profiling specific ACPAs has the potential to improve the diagnosis and stratification of $\mathrm{ACPA}^{+}$RA. Further, we show that a history of cigarette smoking and possession of the HLA-DRB1 SE alleles are associated with the presence and extent of ACPA reactivity in certain anti-CCP ${ }^{-}$RA patients, suggesting that smoking and HLA-DRB1 SE alleles may contribute to the development of $\mathrm{ACPA}$ in anti-CCP ${ }^{+} \mathrm{RA}$ and in anti-CCP ${ }^{-} \mathrm{RA}$. 
Correction notice This article has been corrected since it was published Online First. The affiliation for Michelle Delanoy has been corrected to ' 5 ' (Bio-Rad Laboratories).

Contributors Guarantor of integrity of the entire study: JS and WHR. Study concepts and design: CAW, JS, LJL, CB, SS, LA, MD, TML, RPW, RB, PEC, SRB, LK and WHR. Data collection and interpretation: CAW, JS, LIL and PEC. Statistical analysis: CAW, JS, LL, RB and PEC. Manuscript preparation: CAW, JS, LJL, TML and WHR. Final approval of manuscript: CAW, JS, LJL, CB, SS, LA, MD, TML, RPW, RB, PEC, SRB, LK and WHR.

Funding This work was supported by a National Institutes of Health (NIH) National Institute of Heart, Lung and Blood (NHLBI) Proteomics Center (N01-HV-00242), a NIH National Institute of Allergy and Immunology (NIAID) U01 (Al101981) and Veterans Affairs Health Care System funding to WHR. The work on the Swedish EIRA cohort was supported by grants from the PRIMI programme, and from an ERC senior investigator grant to LK.

Competing interests WHR is an inventor on patents 11/788232 and 12/214670, which are owned by Stanford University and licensed to Bio-Rad Laboratories. All other authors have no disclosures. JS received salary support from an American College of Rheumatology Research and Education Foundation Physician Scientist Development Award and the Department of Veterans Affairs.

Ethics approval This study was conducted with the approval of the institutional review board at Stanford University School of Medicine or the Karolinska Institute.

Provenance and peer review Not commissioned; externally peer reviewed.

Data sharing statement Original datasets will be uploaded to and shared via GEO.

Open Access This is an Open Access article distributed in accordance with the Creative Commons Attribution Non Commercial (CC BY-NC 3.0) license, which permits others to distribute, remix, adapt, build upon this work non-commercially, and license their derivative works on different terms, provided the original work is properly cited and the use is non-commercial. See: http://creativecommons.org/ licenses/by-nc/3.0/

\section{REFERENCES}

1 Schellekens GA, Visser $H$, de Jong BA, et al. The diagnostic properties of rheumatoid arthritis antibodies recognizing a cyclic citrullinated peptide. Arthritis Rheum 2000;43:155-63.

2 Van Wenrooij WJ, van Beers JJ, Pruijn GJ. Anti-CCP antibodies: the past, the present and the future. Nat Rev Rheumatol 2011;7:391-8.

3 Hueber W, Kidd BA, Tomooka BH, et al. Antigen microarray profiling of autoantibodies in rheumatoid arthritis. Arthritis Rheum 2005;52:2645-55.

4 Hueber W, Tomooka BH, Zhao X, et al. Proteomic analysis of secreted proteins in early rheumatoid arthritis: anti-citrulline autoreactivity is associated with up regulation of proinflammatory cytokines. Ann Rheum Dis 2007;66:712-19.

5 Heliövaara M, Aho K, Aromaa A, et al. Smoking and risk of rheumatoid arthritis. J Rheumatol 1993;20:1830-5.

6 Stolt P, Bengtsson C, Nordmark B, et al. Quantification of the influence of cigarette smoking on rheumatoid arthritis: results from a population based case-control study, using incident cases. Ann Rheum Dis 2003;62:835-41.

7 Gregersen PK, Silver J, Winchester RJ. The shared epitope hypothesis. An approach to understanding the molecular genetics of susceptibility to rheumatoid arthritis. Arthritis Rheum 1987;30:1205-13.
8 Klareskog L, Stolt P, Lundberg K, et al. A new model for an etiology of rheumatoid arthritis: smoking may trigger HLA-DR (shared epitope)-restricted immune reactions to autoantigens modified by citrullination. Arthritis Rheum 2006;54:38-46.

9 Monach PA, Hueber W, Kessler B, et al. A broad screen for targets of immune complexes decorating arthritic joints highlights deposition of nucleosomes in rheumatoid arthritis. Proc Natl Acad Sci USA 2009;106:15867-72.

10 Zhao X, Okeke NL, Sharpe 0, et al. Circulating immune complexes contain citrullinated fibrinogen in rheumatoid arthritis. Arthritis Res Ther 2008;10:R94.

11 Mahdi $\mathrm{H}$, Fisher BA, Källberg H, et al. Specific interaction between genotype, smoking and autoimmunity to citrullinated alpha-enolase in the etiology of rheumatoid arthritis. Nat Genet 2009;41:1319-24.

12 Arnett FC, Edworthy SM, Bloch DA, et al. The American Rheumatism Association 1987 revised criteria for the classification of rheumatoid arthritis. Arthritis Rheum 1988;31:315-24.

13 Tibshirani R, Hastie T, Narasimhan B, et al. Diagnosis of multiple cancer types by shrunken centroids of gene expression. Proc Natl Acad Sci USA 2002;99:6567-72.

14 Eisen MB, Spellman PT, Brown PO, et al. Cluster analysis and display of genome-wide expression patterns. Proc Natl Acad Sci USA 1998;95:14863-8.

15 Bizzaro N, Mazzanti G, Tonutti E, et al. Diagnostic accuracy of the anti-citrulline antibody assay for rheumatoid arthritis. Clin Chem 2001;47:1089-93.

16 Stone R, Coppock JS, Dawes PT, et al. Clinical value of ELISA assays for IgM and IgG rheumatoid factors. J Clin Pathol 1987;40:107-11.

17 Sebbag M, Moinard N, Auger I, et al. Epitopes of human fibrin recognized by the rheumatoid arthritis-specific autoantibodies to citrullinated proteins. Eur J Immunol 2006;36:2250-63.

18 Lutteri L, Malaise M, Chapelle JP. Comparison of second- and third-generation anti-cyclic citrullinated peptide antibodies assays for detecting rheumatoid arthritis. Clin Chim Acta 2007;386:76-81.

19 Bizzaro N, Tonutti E, Tozzoli R, et al. Analytical and diagnostic characteristics of 11 2nd- and 3rd-generation immunoenzymatic methods for the detection of antibodies to citrullinated proteins. Clin Chem 2007;53:1527-33.

20 Sghiri R, Bouajina E, Bargaoui D, et al. Value of anti-mutated citrullinated vimentin antibodies diagnosing rheumatoid arthritis. Rheumatol Int 2008;29:59-62.

21 Whiting PF, Smidt N, Steme JA, et al. Systematic review: accuracy of anti-citrullinated peptide antibodies for diagnosing rheumatoid arthritis. Ann Intern Med 2010;152:456-64.

22 Sokolove J, Bromberg R, Deane KD, et al. Autoantibody epitope spreading in the pre-clinical phase predicts progression to rheumatoid arthritis. PLOS ONE 2012;7: e35296.

23 Nielen MM, van Schaardenburg D, Reesink HW, et al. Specific autoantibodies precede the symptoms of rheumatoid arthritis: a study of serial measurements in blood donors. Arthritis Rheum 2004;50:380-6.

24 del Val del Amo N, Ibanez BR, Fito MC, et al. Anti-cyclic citrullinated peptide antibody in rheumatoid arthritis: relation with disease aggressiveness. Clin Exp Rheumatol 2006;24:281-6.

25 Rönnelid J, Wick MC, Lampa J, et al. Longitudinal analysis of citrullinated protein/ peptide antibodies (anti-CP) during 5 year follow up in early rheumatoid arthritis: anti-CP status predicts worse disease activity and greater radiological progression. Ann Rheum Dis 2005;64:1744-9.

26 Vencovský J, Machácek S, Sedová L, et al. Autoantibodies can be prognostic markers of an erosive disease in early rheumatoid arthritis. Ann Rheum Dis 2003:62:427-30.

27 van Dongen $H$, van Aken J, Lard LR, et al. Efficacy of methotrexate treatment in patients with probable rheumatoid arthritis: a double-blind, randomized, placebo-controlled trial. Arthritis Rheum 2007;56:1424-32. 